\title{
Is there a role for computerized electroencephalographic analysis in the diagnosis of cerebral sinus venous thrombosis?
}

\author{
Mojtaba Rismanchi* \\ Shiraz University of Medical Sciences, Shiraz, Iran
}

\begin{abstract}
Computerized electroencephalographic (EEG) analysis yields precise information about the function of the underlying cerebral tissue. This can reveal asymmetry in function or localize possible cerebral lesions. This capability is better appreciated in entities with unsatisfactory diagnostic modalities such as cerebral venous thrombosis (CVT). It is possible to increase the diagnostic precision by combining findings from computerized EEG analysis and brain magnetic resonance imaging. The presence of functional impairment in territories of cerebral venous drainage dictated by cerebral EEG analysis and with normal cerebral imaging makes the diagnosis of cerebral venous thrombosis more probable in a patient suspicious to this diagnosis.
\end{abstract}

\section{Introduction}

Computerized processing of electroencephalographic (EEG) signals can be a promising tool in the localization of cerebral lesions especially for those lesions that the state-of-the-art imaging modalities are not satisfactory, a typical example of which is cerebral venous thrombosis (CVT). This entity is easy to treat but it is otherwise readily fatal that is owing to its various presentations and its difficult imaging interpretation [1]. The typical presentation of CVT is headache that is sometimes impossible to differentiate it from innocent causes of this symptom [2]. CVT results from thrombosis of venous sinuses destabilizing the brain tissue at the level of capillaries. It is shown that CVT is accompanied by increased rate of seizures (approximately $40 \%$ ), focal neurological deficits, altered regional cerebral blood flow and focal cerebral edema [3-5]. All of these abnormalities indicate that subtle abnormal neural firings are highly probable in CVT, though these abnormal electrical activities cannot always be detected by conventional interpretations of EEG. However, subtle EEG abnormalities can be easily detected by various methods of computerized EEG analysis. In this article, the value of brain functional assessment by means of computerized EEG analysis is addressed and the practical approaches in its interpretation is discussed.

\section{Proposed methods}

Using brain symmetry index for the detection of functional impairment as a result of CVT

A good criterion for abnormal electrical behavior as a result of CVT can be derived from the comparison of corresponding left and right contralateral venous territories for lateral sinuses and anteroposterior vertex areas for the study of superior saggital sinus territorial brain tissue in a search for asymmetry of function indicating possible lesion (Figure 1). The recently developed Brain symmetry index (BSI) provides a sensitive criterion for the detection of asymmetry between right and left hemispheres [6,8]. BSI is defined as the absolute value of the relative difference of average spectral density of the right and left hemispheres estimated by Fast Fourier transform in the frequency range of 1 to $25 \mathrm{~Hz}$ (equation 1) [6,7].

$$
\operatorname{BSI}(t)=\frac{1}{M} \sum_{j=1}^{M}\left\|\sum_{i=1}^{N} \frac{R_{i j}(t)-l_{i j}(t)}{R_{i j}(t)+l_{i j}(t)}\right\|
$$

Where $\mathrm{N}$ is the number of channel pairs, $\mathrm{M}$ is the number of Fourier coefficient, with $R_{i j}(t)$ and $l_{i j}(t)$ indicating the corresponding Fourier coefficients in right and left hemispheres. Other more sensitive modifications of this index are described in the literature [8]. BSI provides a numerical criterion for the study of asymmetry of function between hemispheres. BSI equal to 1 denotes maximal asymmetry and 0 denotes perfect symmetry, while in normal individuals BSI is found to be $0.042 \pm 0.005[6,7]$. Although not previously performed, BSI can be used for the detection of asymmetry in corresponding specific areas between right and left hemispheres. This makes it possible to study the corresponding cerebral venous territories in case of CVT.

The venous part of brain vasculature harbors great misleading variations. Therefore, the results of EEG analysis have to be interpreted in accordance with brain magnetic resonance imaging (MRI) and Venography (MRV) in order to find EEG mismatch with anatomical locations of venous system in MRI and MRV. This finding indicates that the asymmetry is due to functional impairment of venous system and not due to other possible focuses of abnormal electrical activity. The interpretation would be that, a symmetric venous system produces asymmetric EEG study, all of which in a patient suspicious to CVT point to the nonfunctional or thrombosed venous segment. It is worth noting that regarding computerized analysis of EEG for detecting CVT, the montage can be modified so that more data acquisition can be performed from the territories of venous drainage (Figure 1) [9].

*Correspondence to: Mojtaba Rismanchi MD, Neurologist, Shiraz University of Medical Sciences, Shiraz, Iran, Tel: 989173073027, E-mail: moj.rismanchi@gmail.com

Received: March 18, 2019; Accepted: May 27, 2019; Published: May 30, 2019 


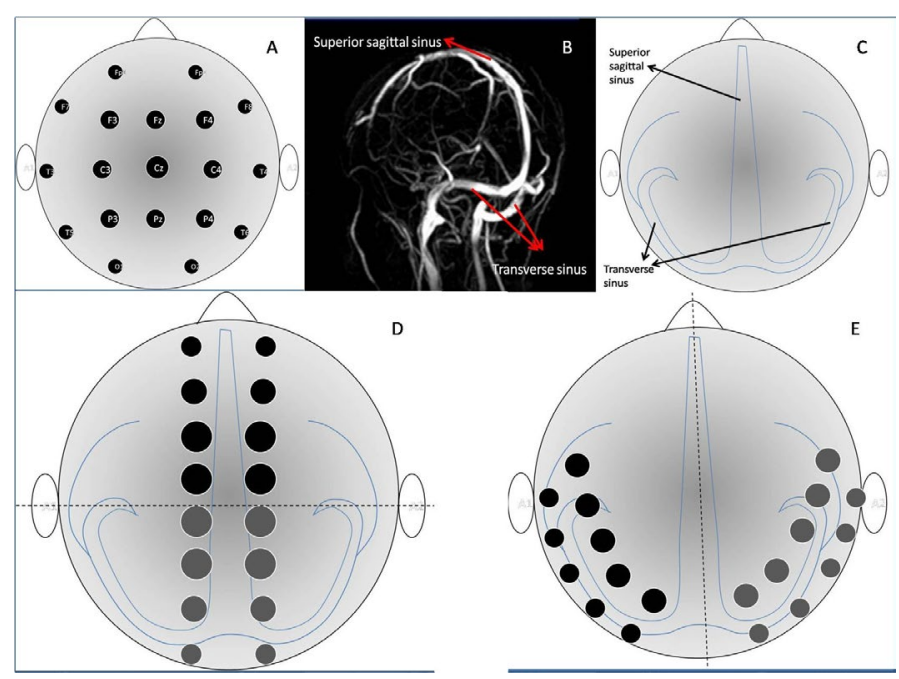

Figure 1. Usual set of EEG leads and its locations in schematic representation (A). Normal cerebral venous structure is represented in a normal MRV(B). Schematic representation of cerebral venous structure in scalp view that is usually used for illustration of EEG leads (C). These include the superior sagittal sinus in the midline and transverse sinuses on two sides. Modified assembly of EEG leads in order to detect the asymmetries in sagittal sinus (D), transverse sinuses (E) and their corresponding cortical veins

\section{Using EEG localization methods in the delineation of functional impairments due to CVT}

It is possible to detect and localize abnormalities in cerebral function Using localization methods in the analysis of EEG. Approximate entropy (ApEn) and slow wave coefficient (SWC) are methods in the field of EEG analysis with the capability to localize the area with abnormal function. ApEn is a nonlinear method to describe the level of complexity and regularity in a time series, thereby measuring the irregulariy of signals (10). SWC provides an indicator of cerebral dysfunction by estimating the predominance of low frequency bands in a tracing of EEG and is defined as:

$$
S W C=\frac{\text { spectrum of }(\delta+\theta)}{\text { spectrum of }(\alpha+\beta)}
$$

Where, $\delta, \theta, \alpha$ and $\beta$ represents EEG frequency band of 1 to $4 \mathrm{~Hz}$, 4.1 to $8 \mathrm{~Hz}, 8.1$ to $13 \mathrm{~Hz}$ and 13.1 to $30 \mathrm{~Hz}$ respectively. The spectra of these frequency bands are extracted from EEG by the implementation of Fourier transform technique. It is shown that brain injury causes increase in slow wave and decrease in fast wave bands in the power spectrum of EEG [10]. Full explanation and the application of these methods can be found in the referring article [10].

The presence of functional abnormalities localized in the territories of cerebral venous drainage along with no underlying structural abnormalities detected by MRI that explain the abnormal firings in a setting of possible CVT, makes the diagnosis of CVT more probable.

\section{Method interpretation}

The study of cerebral function focusing on cortical areas located in the venous territories by means of computerized EEG analysis is advantageous when the diagnosis of CVT is possible and other modalities for the study of venous sinuses including MRI and MRV are unsatisfactory. In order to perform the proposed modality for the diagnosis of CVT, it is recommended to perform MRI as a basis of interpretation. The interpretation of abnormal EEG in CVT can well be established in the context of normal MRI. In fact, when there are multiple old lesions in venous territories, the results of computerized
EEG analysis are not amenable to interpretation. If the EEG analysis reveals asymmetry in cerebral function- the asymmetry has to be localized either by studying the corresponding tracings by a specialized neurologist or by computerized techniques herein proposedcomparison of the area of abnormal function with the corresponding areas in MRV and MRI images points to the irritating nature of the suspected abnormality rather than an innocent finding or artifact. Irritated areas derived from EEG analysis especially when located in venous territories- whether containing lesion or not in MRI imageswith no known underlying pathologies in a patient suspicious of CVT makes this diagnosis probable.

\section{Conclusion}

Herein it is claimed that subtle EEG abnormalities are present in cases of CVT though not detected by conventional EEGs. Furthermore, it is proposed that the combination of computerized EEG analysis as a dynamic diagnostic modality with MRI and MRV as anatomical diagnostic modalities makes it possible to better evaluate cerebral function. This technique would be similar to ventilation perfusion scan for the detection of pulmonary emboli. In both of these techniques a mismatch between two inherently different study modalities makes the presence of thrombosis probable. For further optimization of the proposed method, it is also possible to decrease the size and hence increase the number of computerized EEG study leads.

\section{References}

1. Leach JL, Fortuna RB, Jones BV, Gaskill-Shipley MF (2006) Imaging of cerebral venous thrombosis: Current techniques, spectrum of findings, and diagnostic pitfalls. Radiographics 26: S19-S41.

2. Coutinho JM, Ferro JM, Canhão P, Barinagarrementeria F, Cantú C, et al. (2009) Cerebral venous and sinus thrombosis in women. Stroke 40: 2356-2361.

3. Al-Hashel JY, John JK, Vembu P (2014) Venous thrombosis of the brain. Retrospective review of 110 patients in kuwait. Neurosciences Riyadh, Saudi Arabia 19: 111-117.

4. Kawai N, Shindou A, Masada T, Tamiya T, Nagao S (2005) Hemodynamic and metabolic changes in a patient with cerebral venous sinus thrombosis: Evaluation using o-15 positron emission tomography. Clinical Nuclear Medicine 30: 391-394.

5. Saposnik G, Barinagarrementeria F, Brown RD Jr, Bushnell CD, Cucchiara B, et al. (2011) American heart association stroke council and the council on epidemiology and prevention. Diagnosis and management of cerebral venous thrombosis: a statement for healthcare professionals from the American Heart Association/American Stroke Association. Stroke 42: 1158-1192.

6. Van Putten MJ, Peters JM, Mulder SM, de Haas JA, Bruijninckx CM, et al. (2004) A brain symmetry index (BSI) for online EEG monitoring in carotid endarterectomy. Clinical neurophysiology. 115: 1189-1194.

7. Van Putten MJ, Tavy DL (2004) Continuous quantitative EEG monitoring in hemispheric stroke patients using the brain symmetry index. Stroke 35: 2489-2492.

8. Van Putten MJ (2007) The revised brain symmetry index. Clinical Neurophysiology 118: 2362-2367.

9. Leach JL, Fortuna RB, Jones BV, Gaskill-Shipley MF (2006) Imaging of cerebral venous thrombosis: Current techniques, spectrum of findings, and diagnostic pitfalls 1. Radiographics 26: S19-S41.

10. Yi L, Xiao-ping L, Xian-hong L, Jing-qi L, Wen-wei Y, et al. (2014) Mapping brain injury with symmetrical-channels' EEG signal analysis-a pilot study. Scientific reports 4.

Copyright: (C2019 Rismanchi M. This is an open-access article distributed under the terms of the Creative Commons Attribution License, which permits unrestricted use, distribution, and reproduction in any medium, provided the original author and source are credited. 